\title{
Photorefractive polymeric solitons supported by orientationally enhanced birefringent and electro-optic effects
}

\author{
Fang-Wen Sheu and Ming-Feng Shih \\ Department of Physics, National Taiwan University, Taipei 10617, Taiwan
}

Received April 27, 2000; revised manuscript received September 18, 2000

\begin{abstract}
We predict and analyze the formation of photorefractive polymeric solitons, which are supported by both the orientationally enhanced birefringence and the orientationally enhanced electro-optic effects. The formation conditions and characteristics of this new type of optical spatial soliton are discussed. (C) 2001 Optical Society of America

OCIS codes: 190.5530, 250.5460, 160.5320, 160.5470.
\end{abstract}

\section{INTRODUCTION}

Optical spatial solitons form when the self-focusing effect exactly balances the natural diffraction of the light beam. Kerr-type solitons were the first optical solitons that were predicted, ${ }^{1}$ yet they require very high power and can only be observed in a one-dimensional form and in a slab waveguide owing to both catastrophic self-focusing ${ }^{2}$ and modulation instability. ${ }^{3}$ In the past decade, stable twodimensional optical spatial solitons have already been observed in several saturable nonlinear media. They are the quadratic solitons, which occur during a secondharmonic generation process when the fundamental and the second harmonics are strongly coupled by the secondorder nonlinearities, solitons in saturable nonlinear atomic vapor, which exist at the optical frequency close to the vicinity of the resonant transition, and photorefractive solitons. The discovery ${ }^{4-13}$ of the latest has even loosened the strict conditions for observing optical spatial solitons, i.e., photorefractive solitons require low power (microwatt, or even nanowatt), and may exist in a twodimensional form and in a bulk medium.

Many branches of photorefractive solitons, such as quasi-steady-state photorefractive solitons, ${ }^{4}$ screening solitons,${ }^{5-7}$ photovoltaic solitons, ${ }^{8-10}$ solitons in centrosymmetric photorefractive crystals, ${ }^{11}$ and solitons in photorefractive semiconductors, ${ }^{12}$ have already been discovered. ${ }^{13}$ Among these photorefractive solitons, screening solitons are those most thoroughly studied. It occurs when an externally biasing electric field is partially screened by space charges induced by the soliton light beam. The dc biasing and space-charge field can, by the electro-optic effect, create a waveguidelike refractiveindex profile that focuses the soliton light beam and exactly compensates for the natural diffraction of the soliton light beam.

On the other hand, photorefractive polymers ${ }^{14}$ have begun to attract much research interest since their discovery in 1991. This is because, for quite a few applications (e.g., dynamic holographic storage ${ }^{15}$ and optical information processing ${ }^{16}$ ) that use photorefractive effects, photo- refractive polymers can provide many advantages over the traditional photorefractive inorganic crystals. These advantages include flexibility, cheapness, and ease of processing. Photorefractive polymers ${ }^{17-19}$ of the guest-host type, e.g., PVK:FDEANST:TNF ( poly-N-vinylcarbazole, fluorinated diethylaminonitrostyrene, 2,4,7-trinitro-9fluorenone), DMNPAA:PVK:ECZ:TNF (2,5-dimethyl-4- $p$ nitrophenylazo anisole, poly- $N$-vinylcarbazole, $N$-ethylcarbazole, 2,4,7-trinitro-9-fluorenone) and PVK:PDCST: BBP: $\mathrm{C}_{60}$ （poly- $N$-vinylcarbazole, 4-piperidinobenzylidene-malononitrile, butyl-benzyl-phthalate, $\mathrm{C}_{60}$ ), made from photoconducting polymer hosts and doped with high levels of chromophores with permanent dipole moments and low glass-transition temperature $\left(T_{g}\right)$ are those most successful types of photorefractive polymers. They may provide very high photorefractive efficiency in two-beam coupling or four-wave mixing owing to the orientational enhancement. ${ }^{20}$

Although the major effect causing the index change for the photorefractive polymer is the orientationally enhancement effect of the doped chromophores, which is different from the usual electro-optic effect in the photorefractive inorganic crystals, we showed that the photorefractive polymer can support optical spatial solitons solely by the orientationally enhanced birefringence, and we named them polymeric solitons in a previous paper. $^{21}$ However, except in a few photorefractive polymers whose orientationally enhanced birefringent effect dominates almost all the refractive-index change, the orientationally enhanced electro-optic effect ${ }^{22}$ is not negligible. We therefore need to take the electro-optic effect into account for the formation of polymeric solitons in most polymers.

In this paper we make a complete analysis of the polymeric solitons that are supported by the entire nonlinearity (the orientationally enhanced birefringence plus the orientationally enhanced electro-optic effect) of the chromophores. It is shown that the orientationally enhanced electro-optic effect may counteract or enhance the orientationally enhanced birefringence for giving rise to the 
refractive-index change. If the counteraction is too large, it is not necessary, as concluded in Ref. 21 , that a bright (dark) soliton be supported when its optical field is perpendicular ( parallel) to the applied dc field. Otherwise, only the coefficients of the nonlinear index change is modified owing to the addition of the electro-optic effect. This leaves the soliton-existence curves (the normalized soliton width versus the square root of the solitonintensity ratio) the same as those of the polymeric solitons supported solely by the orientationally enhanced birefringence, but with a new soliton-width unit. In the end, we discuss the formation conditions and the characteristics of the new type of polymeric solitons in several aspects.

\section{GENERAL TREATMENT}

The analysis for polymeric solitons is based on the setup shown in Figs. 1(a) and 2(a). The dc electric field is externally applied in the $x$ direction. The steady-state continuity equation, ${ }^{21}$ rate equations, Gauss's law, and the wave equation for the soliton beam propagating in the $z$ direction are

$$
\begin{aligned}
& J=e \mu \rho E-e D \frac{\partial \rho}{\partial x}=\text { const. } \\
& \frac{\partial N^{-}}{\partial t}=s \phi\left(N-N^{-}\right)\left(I+I_{b}\right)-\frac{e \mu}{\varepsilon_{r}} \rho N^{-}=0, \\
& \frac{\partial T^{+}}{\partial t}=\frac{e \mu}{\varepsilon_{r}} \rho\left(T-T^{+}\right)-r T^{+}=0, \\
& \frac{\partial E}{\partial x}=\frac{4 \pi e}{\varepsilon_{r}}\left(\rho-N^{-}+T^{+}\right), \\
&\left(\frac{\partial^{2}}{\partial x^{2}}+\frac{\partial^{2}}{\partial z^{2}}\right) E_{\mathrm{opt}}+\left(k_{0} n\right)^{2} E_{\mathrm{opt}}=0,
\end{aligned}
$$

where $n$ is the nonlinear index of refraction to be determined. Notice that by the Langevin model ${ }^{18}$ the recombination and trapping rates in Eqs. (2) and (3) are $e \mu / \varepsilon_{r}$. The dependent variables are: $J$, the current density; $E$, the dc field along the $x$ direction, which is screened by the soliton light beam; $\mu,{ }^{23}$ the dc-field dependent hole mobility; $\phi,{ }^{24}$ the dc-field dependent quantum efficiency; $\rho$, the hole density; and $N^{-}$and $T^{+}$, the densities of ionized charge-generating molecules and charge traps, respectively. Relevant parameters are the wave number $k_{0}$ $=2 \pi / \lambda_{0}$, with $\lambda_{0}$ the free-space wavelength of the light; $e$, the charge on a hole; $D$, the diffusion coefficient; $N$ and $T$, the densities of total charge-generating molecules and charge traps, respectively; $s$, the photoexcitation cross section; $\varepsilon_{r}$, the static relative permittivity of the polymers; and $r$, the thermal detrapping rate. The optical field of the soliton beam is $E_{\text {opt }}(x, z)$ $=\varphi(x, z) \exp \left(i k_{0} n_{b} z\right) \sqrt{I_{b}}+$ c.c., which is polarized in the $x$ (or $y$ ) direction, where $\varphi$ is the slowly varying envelope of the soliton normalized to $\sqrt{I_{b}}, I_{b}$ is the background illumination, and $n_{b}$ is the unperturbed refractive index. The actual intensity of the soliton is therefore $I$ $=|\varphi|^{2} I_{b}$, and the soliton-intensity ratio is defined as the maximum of $|\varphi|^{2}$. Utilizing the variable transformation $\xi=x / x_{0}, \zeta=z /\left(2 k_{0} n_{b} x_{0}^{2}\right)$, with $x_{0}$ an arbitrarily chosen normalizing spatial width, $\Delta\left(n^{2}\right)=n^{2}-n_{b}^{2}$, and $\varphi(\xi, \zeta)$ $=u(\xi) \exp (i \Gamma \zeta)$, where $u$ is the normalized soliton amplitude and $\Gamma$ is the soliton propagation constant, we transform Eq. (5) into a dimensionless nonlinear wave equation:

$$
\frac{\mathrm{d}^{2} u(\xi)}{\mathrm{d} \xi^{2}}=u^{\prime \prime}(\xi)=\left[\Gamma-k_{0}^{2} x_{0}^{2} \Delta\left(n^{2}\right)\right] u(\xi) .
$$

With typical photorefractive polymer parameters, ${ }^{19}$ of which $T^{+}$and $N^{-}$of the order of $10^{17} \mathrm{~cm}^{-3}$ are much smaller than $N$ of the order of $10^{19} \mathrm{~cm}^{-3}$ and much larger than $\rho$ of the order of $10^{15} \mathrm{~cm}^{-3}$, under the soliton and background illumination of intensity $0.1 \mathrm{~W} / \mathrm{cm}^{2}$, we have approximation $\rho \cong \varepsilon_{r} s \phi N\left(I+I_{b}\right) / e \mu N^{-}$from Eq. (2)

(a)

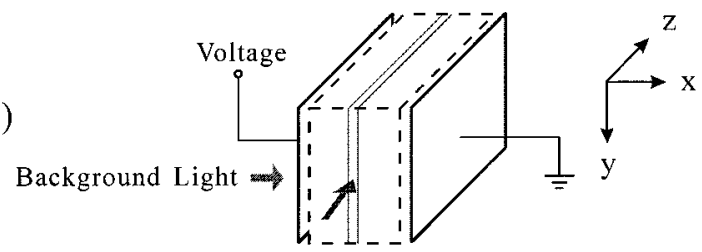

Bright Soliton Light

(b)

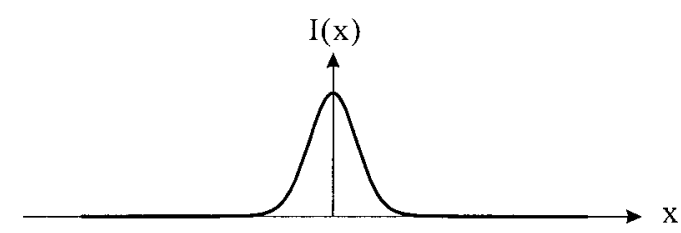

(c)

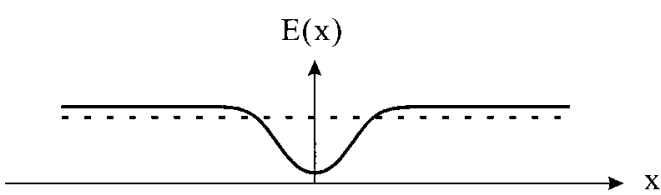

(d)

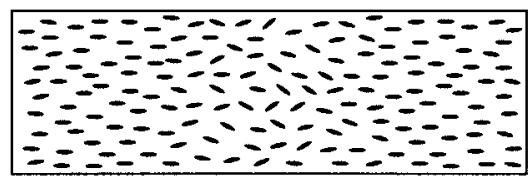

(e)

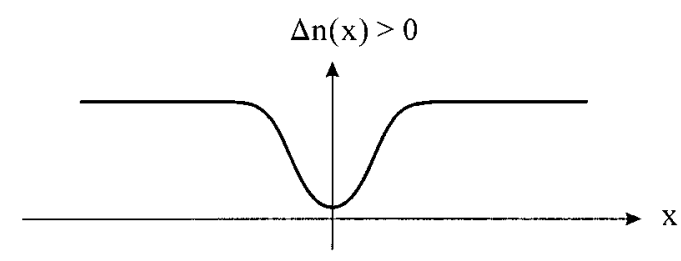

(f)

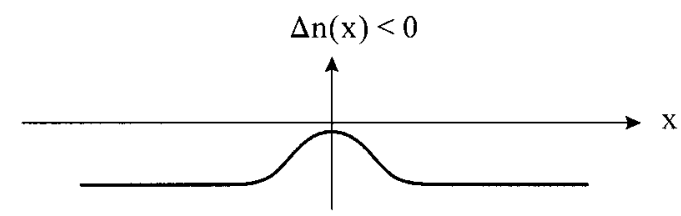

Fig. 1. Formation of bright photorefractive polymeric optical spatial solitons: (a) arrangement; (b) intensity distribution; (c) electric field distribution; (d) the alignment of the chromophores, where the degrees of the alignment are exaggerated and should be more random in a real situation; (e) the index-change profile for $C_{x, y}>0$; (f) the index-change profile for $C_{x, y}<0$. 
(a)

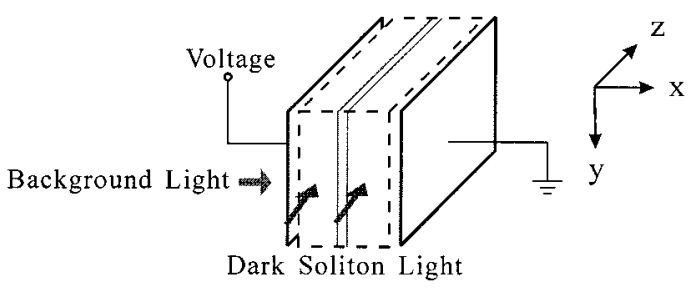

(b)

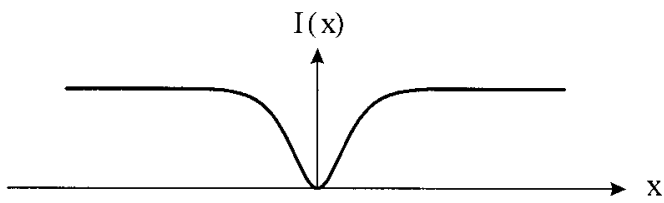

(c)

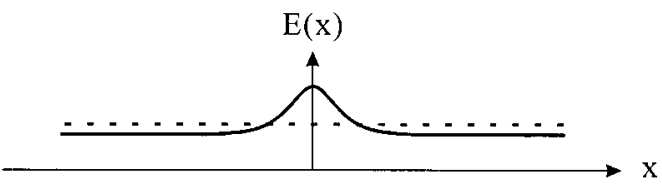

(d)

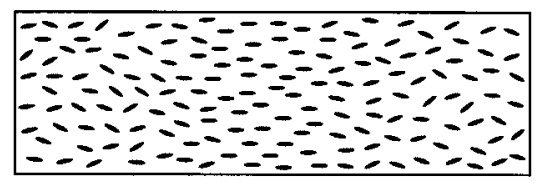

(e)

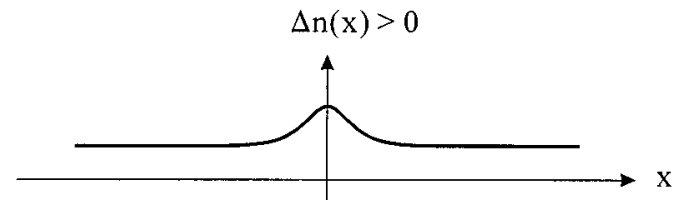

(f)

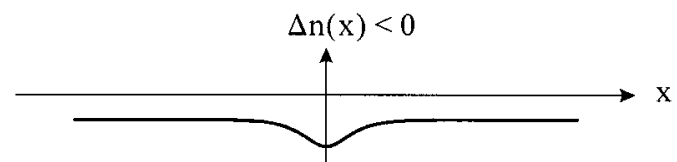

Fig. 2. Formation of dark photorefractive polymeric optical spatial solitons: (a) arrangement; (b) intensity distribution; (c) electric field distribution; (d) the alignment of the chromophores; (e) the index-change profile for $C_{x, y}>0$; (f) the index-change profile for $C_{x, y}<0$.

and $N^{-} \cong T^{+}-\left(\varepsilon_{r} / 4 \pi e\right)(\partial E / \partial x)$ from Eq. (4). We further drop ${ }^{25}$ the term containing $\partial E / \partial x$ and obtain $N^{-}$ $\cong T^{+}$. By assuming that the thermal detrapping is negligible, we have $T^{+} \cong T$ from Eq. (3) and consequently $\rho$ $\cong\left(\varepsilon_{r} s N / e T\right)\left[\phi\left(I+I_{b}\right) / \mu\right]$. We also ignore ${ }^{25}$ the diffusion term in Eq. (1) and obtain $J \cong e \mu \rho E \cong$ const., implying $\phi\left(I+I_{b}\right) E \cong$ const. Using the relationship ${ }^{24} \phi$ $\propto E^{m}$, we easily derive the total dc electric field as a function of the soliton intensity

$$
\begin{aligned}
E^{m+1} & =E_{\infty}^{m+1}\left(I_{\infty}+I_{b}\right) /\left(I+I_{b}\right) \\
& =E_{\infty}^{m+1}\left(I_{\infty}+I_{b}\right) /\left(u^{2} I_{b}+I_{b}\right),
\end{aligned}
$$

where $E_{\infty}$ and $I_{\infty}$ are, respectively, the electric field $E$ and the soliton intensity $I$, far away from the soliton center, at which they are constant.
A. Change of Macroscopic Polarization from Orientationally Enhanced Birefringent and Electro-Optic Effects

To connect Eqs. (6) and (7), we need to obtain the change of the nonlinear refractive index $\Delta\left(n^{2}\right)=n^{2}-n_{b}^{2}$ as a function of $E$, then as a function of $u$. We now focus on the response of the doped nonlinear optical chromophores with large permanent dipole moment and rotational mobility, in the presence of the soliton optical field and the dc field. The microscopic dipole moment of each chromophore molecule is $\mathbf{p}=\boldsymbol{\mu}_{D}+\alpha \cdot \mathbf{E}+\beta: \mathbf{E E}+\cdots$, where $\mu_{D}$ is the molecular permanent dipole moment whose direction is along the molecular c axis, $\alpha$ is the molecular optical polarizability, and $\beta$ is the molecular optical hyperpolarizability tensor. The induced macroscopic polarization at optical frequency $\omega$ is obtained from the orientational averaging of the molecular induced dipole moment:

$$
\begin{aligned}
\mathbf{P}^{\omega}= & N_{\mathrm{ch}}\left\langle\mathbf{p}^{\omega}\right\rangle=N_{\mathrm{ch}}\left\langle\alpha(\omega) \cdot \mathbf{E}^{\omega}\right\rangle \\
& +N_{\mathrm{ch}}\left\langle\beta(-\omega ; 0, \omega): \mathbf{E}^{0} \mathbf{E}^{\omega}\right\rangle+\cdots,
\end{aligned}
$$

where $N_{\text {ch }}$ is the number density of the chromophores, $\mathbf{E}^{0}$ is the dc electric field, and $\mathbf{E}^{\omega}$ is the optical electric field.

For convenience we set the laboratory coordinate axes to be $(x, y, z)$ and the molecular principal axes frame to be $(1,2,3)$ as shown in Fig. 3 . The induced first-order microscopic dipole moment in the frame of the molecular principal axes is $p_{i}^{(1)}=\alpha_{i i}(\omega) E_{i}^{\omega}$, with $i=1,2,3$. Since the chromophores are uniaxial, we set $\alpha_{11}=\alpha_{22}=\alpha_{\perp}$ and $\alpha_{33}=\alpha_{\|}$. The induced microscopic second-order dipole moment is $p_{i}^{(2)}=\beta_{i j k}(-\omega ; 0, \omega) E_{j}^{0} E_{k}^{\omega}$, with $i, j, k$ $=1,2,3$, referring to the principal axes of the molecule. For a rodlike chromophore molecule the hyperpolarizability $\beta_{333}$ is the dominant term. ${ }^{22}$ Hence we assume that the only dominant term is $p_{3}^{(2)} \cong \beta_{333}(-\omega ; 0, \omega) E_{3}^{0} E_{3}^{\omega}$, which is along the molecular 3 -axis. Since the polarity of the dc field is along the $x$ direction, we set $\mathbf{E}^{0}=E_{x}^{0} \hat{x}$.

If we launched the light beam with polarization parallel to the $x$ axis (that is, $\mathbf{E}^{\omega}=E_{x}^{\omega} \hat{x}$ ), the induced first- and

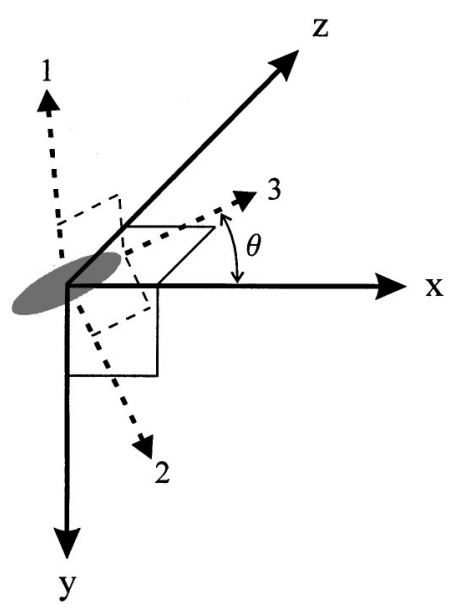

Fig. 3. Solid lines indicate the laboratory coordinates axes $(x, y, z)$. Dashed lines indicate the molecular principal axes $(1$, 2,3 ) with the c axis along the 3 -axis. The dc bias electric field is applied along the $x$ axis. $\theta$ is the angle between the chromophore dipole (3-axis) and the dc poling field ( $x$ axis). This system has a macroscopic rotational symmetry along the $x$ axis. 
second-order macroscopic polarizations at the optical frequency and in the $x$ direction are

$$
\begin{aligned}
P_{x}^{(1), \mathrm{BR}}= & N_{\mathrm{ch}}\left\langle p_{x}^{(1)}\right\rangle \\
= & N_{\mathrm{ch}}\left\langle p_{1}^{(1)} \cos \theta_{1 x}+p_{2}^{(1)} \cos \theta_{2 x}+p_{3}^{(1)} \cos \theta_{3 x}\right\rangle \\
= & N_{\mathrm{ch}}\left\langle\alpha_{\perp} E_{x}^{\omega} \cos ^{2} \theta_{1 x}+\alpha_{\perp} E_{x}^{\omega} \cos ^{2} \theta_{2 x}\right. \\
& \left.+\alpha_{\|} E_{x}^{\omega} \cos ^{2} \theta_{3 x}\right\rangle \\
= & N_{\mathrm{ch}}\left\langle\alpha_{\perp}+\Delta \alpha \cos ^{2} \theta\right\rangle E_{x}^{\omega}, \\
P_{x}^{(2), \mathrm{EO}=} & N_{\mathrm{ch}}\left\langle p_{x}^{(2)}\right\rangle=N_{\mathrm{ch}}\left\langle p_{3}^{(2)} \cos \theta_{3 x}\right\rangle \\
= & N_{\mathrm{ch}}\left\langle\beta_{333} E_{3}^{0} E_{3}^{\omega} \cos \theta_{3 x}\right\rangle \\
= & N_{\mathrm{ch}} \beta_{333}\left\langle\cos ^{3} \theta\right\rangle E_{x}^{0} E_{x}^{\omega},
\end{aligned}
$$

where $\theta_{i x}$ is the angle between the molecular principal $i$ axis and the laboratory $x$ axis. In Eq. (9) the relationship of the direction cosines $\cos ^{2} \theta_{1 x}+\cos ^{2} \theta_{2 x}+\cos ^{2} \theta_{3 x}=1$ and $\Delta \alpha \equiv\left(\alpha_{\|}-\alpha_{\perp}\right)$ has been used. $\theta \equiv \theta_{3 x}$ also is the angle between $\boldsymbol{\mu}_{D}$ and $\mathbf{E}^{0}$. The induced first-order macroscopic polarization $P_{x}^{(1), \mathrm{BR}}$ corresponds to the so-called orientationally enhanced birefringence, and the secondorder term $P_{x}^{(2) \text {,EO }}$ corresponds to the orientationally enhanced electro-optic effect in photorefractive polymers. The net change of the sum of the first two terms of the macroscopic polarization at optical frequency and in the $x$ direction owing to the presence of the dc field $E_{x}^{0}=E$, therefore is

$$
\begin{aligned}
\Delta P_{x}^{\omega}= & {\left[P_{x}^{(1), \mathrm{BR}}+P_{x}^{(2), \mathrm{EO}}\right]_{E_{x}^{0}=E}-\left[P_{x}^{(1), \mathrm{BR}}+P_{x}^{(2), \mathrm{EO}}\right]_{E_{x}^{0}=0} } \\
= & N_{\mathrm{ch}} \Delta \alpha\left(\left\langle\cos ^{2} \theta\right\rangle_{E}-\left\langle\cos ^{2} \theta\right\rangle_{0}\right) E_{x}^{\omega} \\
& +N_{\mathrm{ch}} \beta_{333}\left(\left\langle\cos ^{3} \theta\right\rangle_{E} E\right) E_{x}^{\omega} .
\end{aligned}
$$

Similarly, if we launched the light beam that is polarized parallel to the $y$ axis (that is, $\mathbf{E}^{\omega}=E_{y}^{\omega} \hat{y}$ ), the induced first- and second-order macroscopic polarizations at the optical frequency and in the $y$ direction are

$$
\begin{aligned}
& P_{y}^{(1), \mathrm{BR}}=N_{\mathrm{ch}}\left\langle\alpha_{\perp}+\Delta \alpha \cos ^{2} \theta_{3 y}\right\rangle E_{y}^{\omega}, \\
& P_{y}^{(2), \mathrm{EO}}=N_{\mathrm{ch}} \beta_{333}\left\langle\cos \theta_{3 x} \cos ^{2} \theta_{3 y}\right\rangle E_{x}^{0} E_{y}^{\omega} .
\end{aligned}
$$

The net change of the sum of the first two terms of the macroscopic polarization at optical frequency and in the $y$ direction owing to the presence of the dc field $E_{x}^{0}=E$ is

$$
\begin{aligned}
\Delta P_{y}^{\omega}= & \left.P_{y}^{(1), \mathrm{BR}}+P_{y}^{(2), \mathrm{EO}}\right]_{E_{x}^{0}=E}-\left[P_{y}^{(1), \mathrm{BR}}+P_{y}^{(2), \mathrm{EO}}\right]_{E_{x}^{0}=0} \\
= & -N_{\mathrm{ch}} \Delta \alpha \frac{1}{2}\left(\left\langle\cos ^{2} \theta\right\rangle_{E}-\left\langle\cos ^{2} \theta\right\rangle_{0}\right) E_{y}^{\omega} \\
& +N_{\mathrm{ch}} \beta_{333} \frac{1}{2}\left[\left(\langle\cos \theta\rangle_{E}-\left\langle\cos ^{3} \theta\right\rangle_{E}\right) E\right] E_{y}^{\omega} .
\end{aligned}
$$

We used the statistical equality $\left\langle\cos ^{2} \theta_{3 y}\right\rangle=\left\langle\cos ^{2} \theta_{3 z}\right\rangle$ (owing to the homogeneity in $y$ and $z$ ) and $\left\langle\cos ^{2} \theta_{3 x}\right\rangle$ $+\left\langle\cos ^{2} \theta_{3 y}\right\rangle+\left\langle\cos ^{2} \theta_{3 z}\right\rangle=1$ to simplify Eq. (14).

\section{B. Index Change from dc Poling Field}

The changes of the refractive index for $x$ - and $y$-polarized light beams are therefore governed, respectively, by

$$
\begin{aligned}
\Delta\left(n^{2}\right)_{x}= & 4 \pi N_{\mathrm{ch}}\left[\Delta \alpha\left(\left\langle\cos ^{2} \theta\right\rangle_{E}-\left\langle\cos ^{2} \theta\right\rangle_{0}\right)\right. \\
& \left.+\beta_{333}\left\langle\cos ^{3} \theta\right\rangle_{E} E\right], \\
\Delta\left(n^{2}\right)_{y}= & 4 \pi N_{\operatorname{ch}}\left[-\frac{1}{2} \Delta \alpha\left(\left\langle\cos ^{2} \theta\right\rangle_{E}-\left\langle\cos ^{2} \theta\right\rangle_{0}\right)\right. \\
& \left.+\frac{1}{2} \beta_{333}\left(\langle\cos \theta\rangle_{E}-\left\langle\cos ^{3} \theta\right\rangle_{E}\right) E\right] .
\end{aligned}
$$

Both Eqs. (15) and (16) are functions of the dc field $E$. The statistical average of the $m$ th power of the cosine function in Eqs. (15) and (16) can be evaluated ${ }^{26}$ by the Maxwell-Boltzmann distribution function as

$$
\begin{aligned}
\left\langle\cos ^{m} \theta\right\rangle= & \int_{0}^{\pi} \cos ^{m} \theta \exp \left(\mu_{D} E \cos \theta / k_{B} T_{a}\right) \\
& \times \sin \theta \mathrm{d} \theta / \int_{0}^{\pi} \exp \left(\mu_{D} E \cos \theta / k_{B} T_{a}\right) \sin \theta \mathrm{d} \theta .
\end{aligned}
$$

Although Eqs. (15)-(17) and Eq. (7) can be put into Eq. (6) to solve for the soliton amplitude, the combined equation is actually a highly nonlinear differential-integral equation that is too complicated to solve analytically. Nevertheless, for typical photorefractive guest-host polymers with chromophore dipole moment $\mu_{D}<10 \mathrm{D}$, under $E$ $<100 \mathrm{~V} / \mu \mathrm{m}$ and $T_{a}=300 \mathrm{~K}$, we have $\mu_{D} E / k_{B} T_{a}$ $<0.8$. Thus the statistical average of each power of the cosine function can be approximated ${ }^{22}$ by the first nonzero term of their power series:

$$
\begin{aligned}
\left(\left\langle\cos ^{2} \theta\right\rangle_{E}-\left\langle\cos ^{2} \theta\right\rangle_{0}\right) & \cong(2 / 45)\left(\mu_{D} E / k_{B} T_{a}\right)^{2}, \\
\left\langle\cos ^{3} \theta\right\rangle_{E} & \cong\left(\mu_{D} E / k_{B} T_{a}\right) / 5, \\
\left(\langle\cos \theta\rangle_{E}-\left\langle\cos ^{3} \theta\right\rangle_{E} / 2\right) & \cong\left(\mu_{D} E / k_{B} T_{a}\right) / 15 \\
& \cong\left\langle\cos ^{3} \theta\right\rangle_{E} / 3 .
\end{aligned}
$$

Hence the index changes resulting from the dc field $E$ for $x$ - and $y$-polarized light, respectively, are

$$
\begin{aligned}
\Delta\left(n^{2}\right)_{x} & =\Delta\left(n^{2}\right)_{x}^{\mathrm{BR}}+\Delta\left(n^{2}\right)_{x}^{\mathrm{EO}} \cong\left(C_{x}^{\mathrm{BR}}+C_{x}^{\mathrm{EO}}\right) E^{2} \cong C_{x} E^{2}, \\
\Delta\left(n^{2}\right)_{y} & =\Delta\left(n^{2}\right)_{y}^{\mathrm{BR}}+\Delta\left(n^{2}\right)_{y}^{\mathrm{EO}} \cong\left(C_{y}^{\mathrm{BR}}+C_{y}^{\mathrm{EO}}\right) E^{2} \\
& =\left(-C_{x}^{\mathrm{BR}} / 2+C_{x}^{\mathrm{EO}} / 3\right) E^{2} \equiv C_{y} E^{2},
\end{aligned}
$$

where $C_{x}^{\mathrm{BR}}=(2 / 45)\left(4 \pi N_{\mathrm{ch}}\right) \Delta \alpha\left(\mu_{D} / k_{B} T_{a}\right)^{2}, C_{x}^{\mathrm{EO}}=(1 / 5)$ $\times\left(4 \pi N_{\mathrm{ch}}\right) \beta_{333}\left(\mu_{D} / k_{B} T_{a}\right), \quad C_{y}^{\mathrm{BR}}=-C_{x}^{\mathrm{BR}} / 2, \quad$ and $\quad C_{y}^{\mathrm{EO}}$ $=C_{x}^{\mathrm{EO}} / 3$. For typical photorefractive polymers, $C_{x}=C_{x}^{\mathrm{BR}}+C_{x}^{\mathrm{EO}}$ is positive, but $C_{y}=-C_{x}^{\mathrm{BR}} / 2+C_{x}^{\mathrm{EO}} / 3$ may be positive or negative. ${ }^{20}$ The larger is the dc field $E$, the more are the chromophores oriented in the $x$ direction. This increases the refractive index for $x$-polarized light and reduces the index for $y$-polarized light when only birefringence is considered. Therefore the nonlinearity $\left(C_{x}\right.$ and $C_{y}$ ) owing to the orientationally enhanced birefringence $\left(C_{x}^{\mathrm{BR}}\right.$ and $\left.-C_{x}^{\mathrm{BR}} / 2\right)$ is positive for $x$-polarized light but negative for $y$-polarized light. However, it is not so for the index change owing to the orientationally enhanced electro-optic effect. This is because $\beta_{333}$ is the only dominant hyperpolarizability term ${ }^{22}$; its contributions to the index changes for $x$ - and $y$-polarized light are 
both positive. As a result, the addition of the electrooptic effect enhances the nonlinearity for $x$-polarized light but reduces the nonlinearity or even reverses the sign for $y$-polarized light.

\section{Wave Equation for Soliton Envelope}

Reduced from Eqs. (6), (7), (18), and (19), the solitonenvelope equation then becomes

$$
u^{\prime \prime}(\xi)=\left\{\Gamma-k_{0}^{2} x_{0}^{2} C_{x, y} E_{\infty}^{2}\left[\frac{u_{\infty}^{2}+1}{u^{2}(\xi)+1}\right]^{2 /(m+1)}\right\} u(\xi),
$$

with $u_{\infty}^{2}=u^{2}(\infty)=I_{\infty} / I_{b}$. We choose the characteristic length $x_{0}=1 /\left(k_{0} E_{\infty} \sqrt{\left|C_{x, y}\right|}\right)$ and simplify Eq. (20) to

$$
u^{\prime \prime}(\xi)=\left\{\Gamma \pm\left[\frac{u_{\infty}^{2}+1}{u^{2}(\xi)+1}\right]^{2 /(m+1)}\right\} u(\xi)
$$

where the plus (minus) sign corresponds to the negative (positive) $C_{x, y}$ value.

We may intuitively understand which sign in Eq. (21) is necessary for forming bright or dark solitons. Let a bright light beam propagate in the biased photorefractive polymer, as shown in Fig. 1(a). The space-charge field induced by the bright light beam then screens the uniform (dotted line) external dc field, giving rise to the total dc field $E$ as shown in Fig. 1(c). As a result of nonuniform chromophore-dipoles reorientation, the index-change profile owing to this dc field is $\Delta\left(n^{2}\right)_{x, y} \cong C_{x, y} E^{2}$. If $C_{x, y}$ is positive, the index change is also positive. Thus the index profile has a local minimum at the beam center [Fig. 1(e)] that may defocus the light beam. On the other hand, if $C_{x, y}$ is negative, the index change is also negative. The resultant index profile [Fig. 1(f)] thus forms a waveguide that may guide the bright light beam itself. We thus conclude that negative $C_{x, y}$ is a necessary condition for bright solitons to form regardless of the polarization of the light beam. Similarly, positive $C_{x, y}$ is necessary for dark solitons to form, as illustrated in Fig. 2. Therefore from Eqs. (18) and (19) we know that if the orientationally enhanced birefringence is the major effect, then $C_{x} \cong C_{x}^{\mathrm{BR}}, C_{y} \cong-C_{x}^{\mathrm{BR}} / 2$, and dark (bright) solitons are supported for the $x(y)$ polarized light beam. Oppositely, if the electro-optic effect dominates, then $C_{x}$ $\cong C_{x}^{\mathrm{EO}}, C_{y} \cong C_{x}^{\mathrm{EO}} / 3$, and only dark solitons, polarized in either the $x$ or the $y$ direction, can be supported for $C_{x}^{\mathrm{EO}}$ $>0$. In one special case, $C_{y}=0=-C_{x}^{\mathrm{BR}} / 2+C_{x}^{\mathrm{EO}} / 3$, the material becomes linear and cannot support any solitons for the $y$-polarized light beam.

As we compare the polymeric solitons supported by the orientationally enhanced birefringence plus the electrooptic effect to the solitons ${ }^{21}$ supported solely by the orientationally enhanced birefringence, the addition of the electro-optic effect does not affect the dimensionless nonlinear soliton-envelope Eq. (21) but only modifies the nonlinear index-change coefficients $C_{x, y}$ and rescales the characteristic length $x_{0}$. The normalized soliton envelopes and the soliton-existence curves therefore are kept unchanged but with a new length unit. Nevertheless for completeness and convenience we still present them here.

\section{Bright Solitons}

The boundary conditions of bright solitons are (i) $u(0)$ $=u_{0}, u^{\prime}(0)=0$, (ii) $u(\infty)=u^{\prime}(\infty)=u^{\prime \prime}(\infty)=0$, and (iii) $u^{\prime \prime}(0) / u(0)<0$. With $u_{\infty}=u(\infty)=0$, Eq. (21) becomes

$$
u^{\prime \prime}=\left[\Gamma+\left(\frac{1}{u^{2}+1}\right)^{2 /(m+1)}\right] u .
$$

Using quadrature and boundary condition (i), we obtain

$$
\begin{aligned}
{\left[u^{\prime}(\xi)\right]^{2}=} & \Gamma\left[u^{2}(\xi)-u_{0}^{2}\right] \\
& +\frac{m+1}{m-1}\left\{\left[u^{2}(\xi)+1\right]^{(m-1) /(m+1)}\right. \\
& \left.-\left[u_{0}^{2}+1\right]^{(m-1) /(m+1)}\right\} \quad \text { for } m \neq 1, \\
{\left[u^{\prime}(\xi)\right]^{2}=} & \Gamma\left[u^{2}(\xi)-u_{0}^{2}\right]+\ln \left[u^{2}(\xi)+1\right] \\
& -\ln \left(u_{0}^{2}+1\right) \quad \text { for } m=1 .
\end{aligned}
$$

Putting boundary conditions (ii) into Eq. (23), we obtain

$$
\begin{aligned}
\Gamma= & \frac{m+1}{m-1}\left[1-\left(u_{0}^{2}+1\right)^{(m-1) /(m+1)}\right] / u_{0}^{2} \\
& \text { for } m \neq 1, \\
\Gamma= & -\ln \left(u_{0}^{2}+1\right) / u_{0}^{2} \quad \text { for } m=1,
\end{aligned}
$$

where $u_{0}^{2}=u^{2}(0)=I(0) / I_{b}$ is the soliton-intensity ratio. The soliton profiles $u(\xi)$ for various values of $u_{0}$ and $m$ can be obtained easily by putting $\Gamma$ into Eq. (22) and solving it numerically. Several examples are shown in Fig.
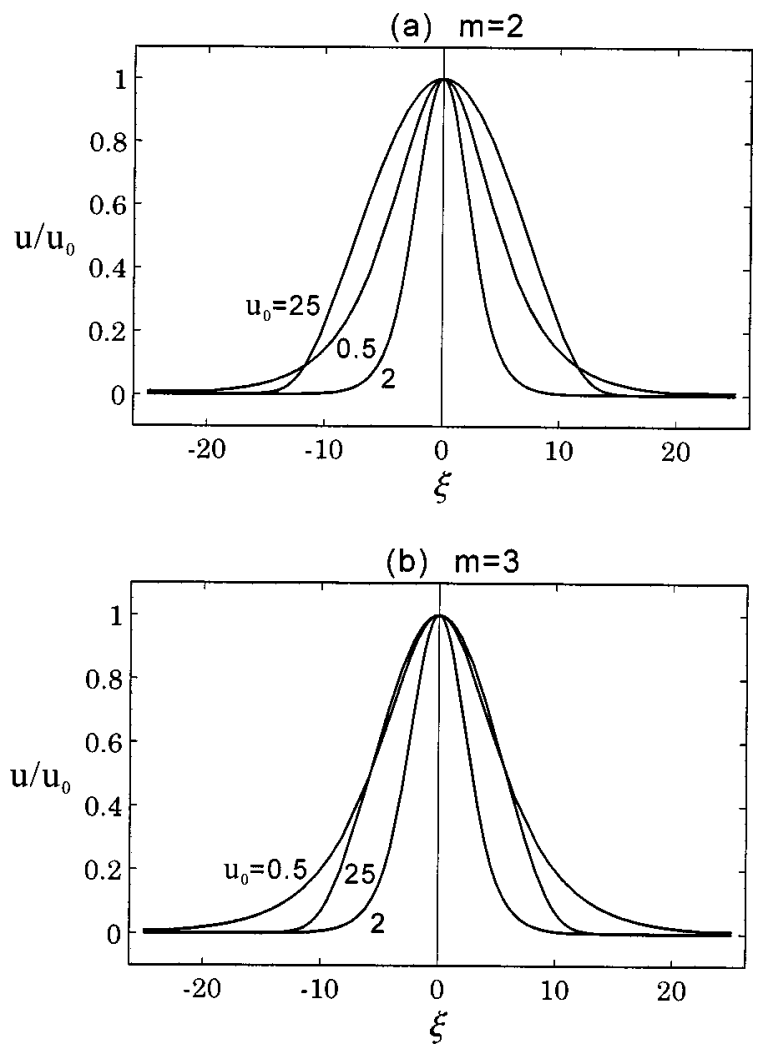

Fig. 4. Normalized soliton profiles $u(\xi) / u_{0}$ of bright photorefractive polymeric optical spatial solitons, for several $u_{0}$ values, and for (a) $m=2$ and (b) $m=3$. 
4. The normalized soliton width $\Delta \xi$, defined as the full width at half-maximum (FWHM) of the normalized soliton intensity $u^{2}(\xi)$, can be evaluated by $\Delta \xi=2 \int_{0}^{\Delta \xi / 2} \mathrm{~d} \xi$ $=2 \int_{u_{0}}^{u_{0} / \sqrt{2}} \mathrm{~d} u / u^{\prime}$, where $u^{\prime}$ is obtained from Eq. (23):

$$
\begin{gathered}
u^{\prime}=\left\{\Gamma\left(u^{2}-u_{0}^{2}\right)+\frac{m+1}{m-1}\left[\left(u^{2}+1\right)^{(m-1) /(m+1)}\right.\right. \\
\left.\left.-\left(u_{0}^{2}+1\right)^{(m-1) /(m+1)}\right]\right\}^{1 / 2} \text { for } m \neq 1, \\
u^{\prime}=\left[\Gamma\left(u^{2}-u_{0}^{2}\right)+\ln \left(u^{2}+1\right)-\ln \left(u_{0}^{2}+1\right)\right]^{1 / 2} \\
\text { for } m=1 .
\end{gathered}
$$

The normalized soliton widths $\Delta \xi$ as a function of $u_{0}$ (existence curves of solitons) for various $m$ are given in Fig. 5.

\section{Dark Solitons}

The boundary conditions for dark solitons are (i) $u(\infty)$ $=u_{\infty}, u^{\prime}(\infty)=u^{\prime \prime}(\infty)=0$, (ii) $u(0)=0$, and (iii) $u^{\prime}(0)$ is real and nonzero. Putting $u^{\prime \prime}(\infty)=0$ and $u(0)=0$ into Eq. (21), we obtain $\Gamma=1$ for all cases, independent of $m$ and $u_{\infty}$. This implies that all dark solitons in a photorefractive polymer propagate at one group velocity (determined solely by bare index perturbation) regardless of their intensity ratio and quantum efficiency. Equation (21) then becomes

$$
u^{\prime \prime}=\left[1-\left(\frac{u_{\infty}^{2}+1}{u^{2}+1}\right)^{2 /(m+1)}\right] u .
$$

The soliton profiles $u(\xi)$ for various values of $u_{\infty}$ and $m$ can be solved numerically. In Fig. 6 we show several examples. Similarly, the normalized width $\Delta \xi$ for dark solitons can be evaluated as well by $\Delta \xi=2 \int_{0}^{u_{\infty} / \sqrt{2}} \mathrm{~d} u / u^{\prime}$, where $u^{\prime}$ is obtained from Eq. (26) by quadrature and boundary condition (ii):

$$
\begin{aligned}
u^{\prime}= & \left\{u^{2}-\frac{m+1}{m-1}\left(u_{\infty}^{2}+1\right)^{2 /(m+1)}\right. \\
& \left.\times\left[\left(u^{2}+1\right)^{(m-1) /(m+1)}-1\right]+\left[u^{\prime}(0)\right]^{2}\right\}^{1 / 2}
\end{aligned}
$$

for $m \neq 1$,

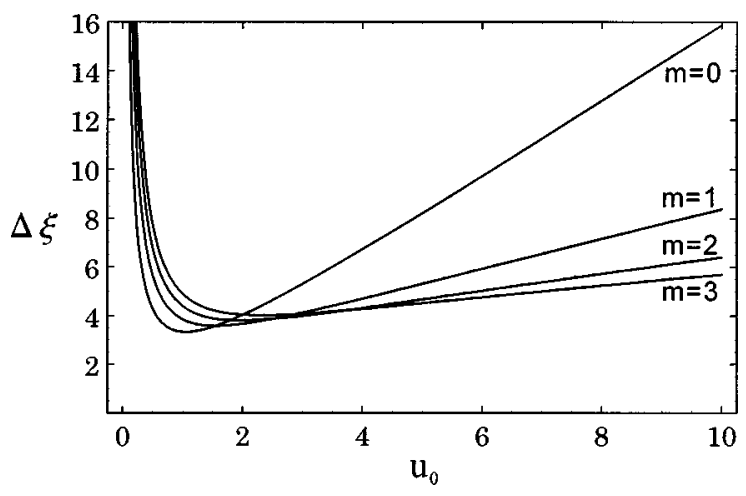

Fig. 5. Normalized soliton widths $\Delta \xi$ of bright photorefractive polymeric optical spatial solitons, as a function of $u_{0}$, for several $m$ values.
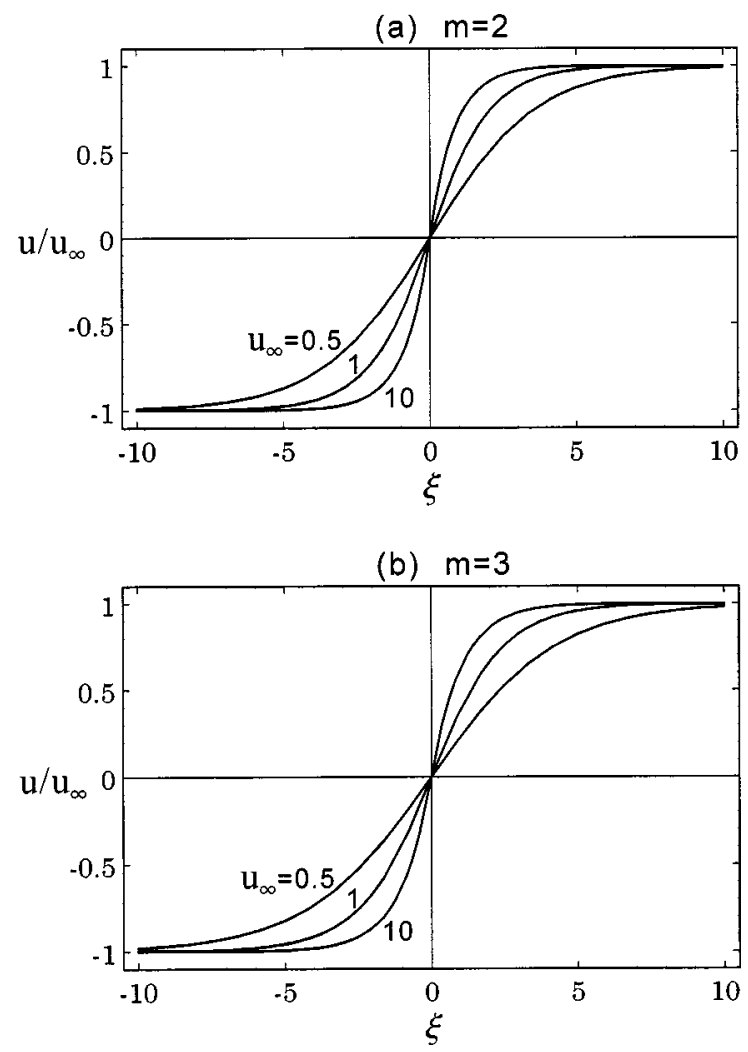

Fig. 6. Normalized soliton profiles $u(\xi) / u_{\infty}$ of dark photorefractive polymeric optical spatial solitons, for several $u_{\infty}$ values, and for (a) $m=2$ and (b) $m=3$.

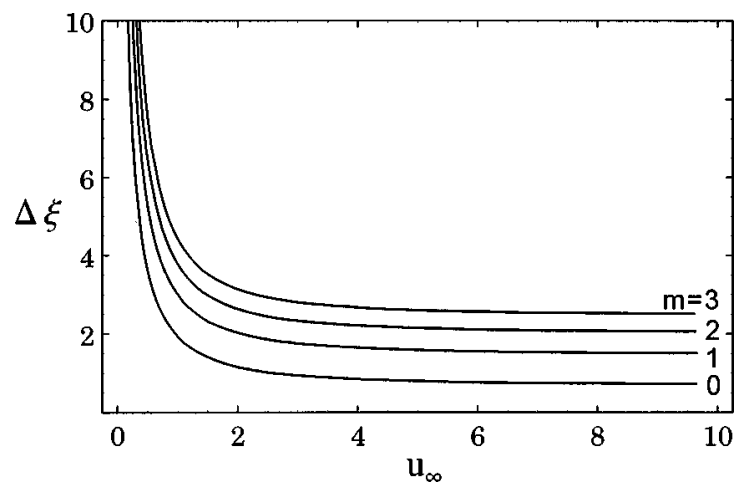

Fig. 7. Normalized soliton widths $\Delta \xi$ of dark photorefractive polymeric optical spatial solitons, as a function of $u_{\infty}$, for several $m$ values.

$$
\begin{array}{r}
u^{\prime}=\left\{u^{2}-\left(u_{\infty}^{2}+1\right) \ln \left(u^{2}+1\right)+\left[u^{\prime}(0)\right]^{2}\right\}^{1 / 2} \\
\text { for } m=1 .
\end{array}
$$

Substituting $\xi \rightarrow \infty$ and $u^{\prime}(\infty)=0$ into Eq. (27), we obtain the first derivative function value of $u$ at $\xi=0$ :

$$
\begin{aligned}
{\left[u^{\prime}(0)\right]^{2}=} & \frac{m+1}{m-1}\left(u_{\infty}^{2}+1\right)^{2 /(m+1)} \\
& \times\left[\left(u_{\infty}^{2}+1\right)^{(m-1) /(m+1)}-1\right]-u_{\infty}^{2} \\
& \text { for } m \neq 1, \\
{\left[u^{\prime}(0)\right]^{2}=} & \left(u_{\infty}^{2}+1\right) \ln \left(u_{\infty}^{2}+1\right)-u_{\infty}^{2} \text { for } m=1 .
\end{aligned}
$$


Hence according to the results of Eqs. (27) and (28), we find that the normalized soliton width $\Delta \xi$ is explicitly a function of $u_{\infty}$, which is the square root of the intensity ratio for dark solitons. The normalized soliton widths $\Delta \xi$ as a function of $u_{\infty}$ for various $m$ are given in Fig. 7 .

\section{DISCUSSION}

An effort to experimentally demonstrate photorefractive polymeric optical spatial solitons is currently being carried out in our laboratory. Although no conclusive result is yet obtained, we would like to address several issues about the formation conditions and characteristics of this new type of optical spatial solitons.

\section{A. Soliton Stability}

Because the nonlinearity forms of polymeric solitons are very similar to the form of screening solitons, especially $^{6,7,11,21}$ for $m=0$ and $m=1$, we do not analyze the stability of polymeric solitons but only borrow the existing stability analysis results from the photorefractive screening solitons and postulate that they have similar stability properties. Nevertheless, it will be our interest to investigate how the power dependence $m$ of the quantum efficiency $\phi$ on the dc field $E$ affects the stability of optical spatial solitons in photorefractive polymer.

\section{B. Material Stability}

In order to support optical spatial solitons, the photorefractive polymer is doped with chromophores that have sufficient permanent dipole moment and rotational mobility. The wavelength dependence of the photostability of nonlinear optical chromophores should be surveyed for deciding the soliton wavelength to prevent photochemical reaction and photodamage of chromophore molecules. ${ }^{27}$ For popular chromophores the suitable wavelength is roughly between 0.8 and $1.3 \mu \mathrm{m}$.

\section{Voltage}

Once we have launched a bright or dark optical beam with known peak intensity, soliton width $\Delta x$, and background illumination $I_{b}$, we can find out the corresponding normalized soliton width $\Delta \xi$ from the soliton-existence curves (Figs. 5 and 7) and evaluate the corresponding field $E_{\infty}$. For optical spatial solitons formed in bulk photorefractive inorganic crystals, ${ }^{7}$ the required voltages are usually estimated by $V \cong E_{\circ} l$, where $l$ is the crystal thickness (electrode separation) because the soliton width is much smaller than the crystal thickness. However, the thickness of photorefractive polymer thin film is usually about tens to hundreds of micrometers, which is not much larger than the soliton width. Hence the required external dc bias voltage across the photorefractive polymer thin film must be evaluated by integrating $E$ $=E_{\infty}\left[\left(u_{\infty}^{2}+1\right) /\left(u^{2}+1\right)\right]^{1 /(m+1)}$ across the actual polymer thickness.

\section{Soliton Size}

The soliton size $\Delta x$ is decided by the so-called intensity ratio and its mapping on the soliton-existence curves (Figs. 5 and 7), whose vertical axis is the normalized soliton size $\Delta \xi$ in the unit $x_{0}=1 /\left(k_{0} E_{\infty} \sqrt{\left|C_{x, y}\right|}\right)$. As a result, the stronger the photorefractive polymeric optical nonlinearity, the smaller both the characteristic length $x_{0}$ and the obtainable minimum soliton size $\Delta x=\Delta \xi x_{0}$. Using the photorefractive polymer parameters ${ }^{14,28} \mathrm{~m}=2.0, n_{b}$ $\cong 1.6$, and $\Delta n_{x} \cong 1.3 \times 10^{-2}$ under external field $E_{\infty}$ $=100 \mathrm{~V} / \mu \mathrm{m}$, with $\lambda_{0}=700 \mathrm{~nm}$, we obtain a minimum soliton width $\Delta x$ of less than $1.5 \mu \mathrm{m}$ for dark solitons or 3 $\mu \mathrm{m}$ for bright solitons if the intensity ratio is adjusted to correspond to the minimum normalized soliton width $\Delta \xi$. Nevertheless, for solitons of this size, which approaches the optical wavelength, the validity of the paraxial approximation, ${ }^{29}$ the elimination of the nonlinear response of the relative permittivity, $\varepsilon_{r}$, and the drop of the diffusion and $\partial E / \partial x$ terms in Eqs. (1) and (4) need to be reinvestigated.

\section{E. Background Illumination}

The desired polarization of the background illumination is selected to meet the smallest electro-optic coefficient. Nevertheless, in a photorefractive polymer, the launching of the background illumination should be arranged differently than that of the photorefractive screening solitons. ${ }^{7}$ As shown in Figs. 1(a) and 2(a), the soliton light beam is incident into the polymer thin film, propagates in the direction perpendicular to that of the dc bias field, and is polarized in the direction either parallel or perpendicular to that of the dc bias field. If the uniform background illumination copropagates with the soliton light beam and is chosen to be linearly polarized in the direction perpendicular to that of the soliton light beam (to avoid interference effects), it still sees the soliton-induced refractiveindex waveguide or antiwaveguide and thus will focus or defocus during the propagation in the polymer. As a result, the uniformity of the background illumination and the constancy of the intensity ratio cannot be maintained. The appropriate way to launch the background illumination therefore is to let it go vertically through the polymer thin film from either side of the transparent indium tin oxide electrodes, and to polarize it in the direction of the soliton propagation. To keep the soliton-intensity ratio constant in the polymer under this configuration, the background illumination from either side of polymer thin film must be suitably linearly attenuated along the propagation of the soliton, for the sake of the absorption of the soliton light beam.

\section{F. Formation Time and Temperature}

Right after turn-on of the external poling electric field, the doped nonlinear optical chromophore dipoles begin the rotational-diffusion process to reach the minimum Helmholtz free-energy configuration. It is found that the time constants associated with the molecular transient reorientation process are not identical for the first- and second-order optical processes. ${ }^{22}$ On the removal of the dc poling field, the guest-host system undergoes another diffusion process of molecular alignment to reach the maximum-entropy state. In the formation process of photorefractive polymeric solitons, the molecular dipoles are originally poled by the external dc bias electric field. After the optical beam is launched, it produces a local space-charge field to screen the external poling field, and the total electric field distribution creates a waveguide- 
like refractive-index profile to guide the optical beam by the orientationally enhanced birefringence and the orientationally enhanced electro-optic effect. Hence the transience of formation of solitons is strongly associated with the light-induced turn-off behavior of the dc poling field in the spatial region of the optical beam, which is governed by charge generation, charge transport, and chromophore reorientation. ${ }^{30}$ In the process of chromophore reorientation, the orientationally enhanced birefringent and electro-optic effects are found to relax in time with different decay time constants, $1 / 6 D$ and $1 / 2 D,{ }^{22}$ respectively, where $D$ is the diffusion constant, so the first-order linear optical process (birefringence) is dominant in the formation of solitons. However, it is also found that the chromophore orientational response of only $490 \mu$ s does not limit the index-grating formation with a fast time constant of $4 \mathrm{~ms}$ in the four-wave-mixing experiments. ${ }^{30}$ That is to say, the reorientation of chromophore dipoles subjected to the nonuniformly screened dc field is much faster than the formation of the soliton-induced spacecharge field. Thus the photoinduced charge generation and charge transport are the major processes that influence the transient photorefractive effect. The formation time is therefore roughly inversely proportional to the local optical intensity, as is the case in photorefractive inorganic crystals. ${ }^{5,7}$ Although the reorientation of the chromophore dipoles is a thermodynamic process that is affected by temperature, by the above arguments we know it has little to do with the formation time of the optical spatial solitons in a photorefractive polymer. However, since the coefficients for index change, $C_{x, y}^{\mathrm{BR}} \propto 1 / T_{a}^{2}$ and $C_{x, y}^{\mathrm{EO}} \propto 1 / T_{a}$, at higher temperature, the required voltage to form a soliton of fixed size is larger.

\section{CONCLUSION}

We have shown that photorefractive polymers doped with nonlinear optical chromophores that have sufficient permanent dipole moment and rotational mobility may support optical spatial solitons. The waveguidelike refractive-index profile comes from the orientationally enhanced birefringent and electro-optic effects of the chromophore dipoles subjected to a screened dc electric field. The formation conditions for these photorefractive polymeric optical spatial solitons and their characteristics are discussed.

The sample preparation of photorefractive polymer and the experimental observation of photorefractive polymeric optical spatial solitons are being carried out at National Taiwan University. We point out that the research of optical spatial solitons in photorefractive polymer merits further investigations, including the dynamical behavior and stability of photorefractive polymeric solitons, improvement of chromophore structures and characterization $^{28,31,32}$ and their effects on generating optical spatial solitons, and applications in integrated and nonlinear optical devices. ${ }^{33}$

\section{ACKNOWLEDGMENT}

This research is supported by the National Science Council, Taiwan, under contract NSC-89-2113-M-002-034. The e-mail address for M.-F. Shih is mfshih@phys.ntu.edu.tw.

\section{REFERENCES AND NOTES}

1. R. Y. Chiao, E. Garmire, and C. H. Townes, "Self-trapping of optical beams," Phys. Rev. Lett. 13, 479-482 (1964).

2. P. L. Kelley, "Self-focusing of optical beams," Phys. Rev. Lett. 15, 1005-1008 (1965).

3. V. E. Zakharov and A. M. Rubenchik, "Instability of waveguides and solitons in nonlinear media," Sov. Phys. JETP 38, 494-500 (1974).

4. M. Segev, B. Crosignani, A. Yariv, and B. Fischer, "Spatial solitons in photorefractive media," Phys. Rev. Lett. 68, 923-926 (1992).

5. M. Segev, G. C. Valley, B. Crosignani, P. Diporto, and A. Yariv, "Steady-state spatial screening solitons in photorefractive materials with external applied field," Phys. Rev. Lett. 73, 3211-3214 (1994).

6. D. N. Christodoulides and M. Carvalho, "Bright, dark, and gray spatial soliton states in photorefractive media," J. Opt. Soc. Am. B 12, 1628-1633 (1995).

7. M. Segev, M. Shih, and G. C. Valley, "Photorefractive screening solitons of high and low intensity," J. Opt. Soc. Am. B 13, 706-718 (1996).

8. G. C. Valley, M. Segev, B. Crosignani, A. Yariv, M. M. Fejer, and M. C. Bashaw, "Dark and bright photovoltaic spatial solitons," Phys. Rev. A 50, R4457-R4460 (1994).

9. M. Taya, M. Bashaw, M. M. Fejer, M. Segev, and G. C. Valley, "Observation of dark photovoltaic spatial solitons," Phys. Rev. A 52, 3095-3100 (1995).

10. M. Segev, G. C. Valley, M. C. Bashaw, M. Taya, and M. M. Fejer, "Photovoltaic spatial solitons," J. Opt. Soc. Am. B 14, 1772-1781 (1997).

11. M. Segev and A. J. Agranat, "Spatial solitons in centrosymmetric photorefractive media," Opt. Lett. 22, 1299-1301 (1997).

12. M. Chauvet, S. Hawkins, G. Salamo, M. Segev, D. Bliss, and G. Bryant, "Self-trapping of planar optical beams by use of the photorefractive effect in InP:Fe," Opt. Lett. 21, 1333-1335 (1996); "Self-trapping of two-dimensional optical beams and light-induced waveguiding in photorefractive InP at telecommunication wavelengths," Appl. Phys. Lett. 70, 2499-2501 (1997).

13. M. Segev and G. Stegeman, "Self-trapping of optical beams: spatial solitons," Phys. Today 51(8), 42-48 (1998), and references therein.

14. S. Ducharme, J. C. Scott, R. J. Twieg, and W. E. Moerner, "Observation of the photorefractive effect in a polymer," Phys. Rev. Lett. 66, 1846-1849 (1991).

15. C. Poga, P. Lundquist, V. Lee, R. Shelby, R. Twieg, and D. Burland, "Polysiloxane-based photorefractive polymers for digital holographic data storage," Appl. Phys. Lett. 69, 1047-1049 (1996)

16. P. Günter and J.-P. Huignard, Photorefractive Materials and Their Applications (Springer, Berlin, 1988 and 1989); Vols. 1 and 2 .

17. K. Meerholz, B. L. Volodin, Sandalphon, B. Kippelen, and N. Peyghambarian, "A photorefractive polymer with high optical gain and diffraction efficiency near 100\%," Nature (London) 371, 497-500 (1994); M. Liphardt, A. Goonesekera, B. Jones, S. Ducharme, J. Takacs, and L. Zhang, "High-performance photorefractive polymers," Science 263, 367-369 (1994).

18. W. E. Moerner and S. M. Silence, "Polymeric photorefractive materials," Chem. Rev. 94, 127-155 (1994).

19. A. Grunnet-Jepsen, C. L. Thompson, R. J. Twieg, and W. E. Moerner, "High performance photorefractive polymer with improved stability," Appl. Phys. Lett. 70, 1515-1517 (1997).

20. W. E. Moerner, S. M. Silence, F. Hache, and G. C. Bjorklund, "Orientationally enhanced photorefractive effect in polymers,” J. Opt. Soc. Am. B 11, 320-330 (1994).

21. M. Shih and F. Sheu, "Photorefractive polymeric optical spatial solitons," Opt. Lett. 24, 1853-1855 (1999).

22. J. W. Wu, "Birefringent and electro-optic effects in poled polymer films: steady-state and transient properties," J. Opt. Soc. Am. B 8, 142-152 (1991).

23. By J. X. Mack, L. B. Schein, and A. Peled, "Hole mobilities 
in hydrazone-polycarbonate dispersions," Phys. Rev. B 39, 7500-7508 (1989), hole mobility $\mu \propto \exp [C(\sqrt{E}-1)]$, where $C$ is an experimentally determined constant.

24. By Onsager model [P. J. Melz, "Photogeneration in trinitrofluorenone-poly( $n$-vinylcarbazole)," J. Chem. Phys. 57, 1694-1699 (1972)], quantum efficiency $\phi \propto E^{m}$, for the static dc field $E$ between 10 and $100 \mathrm{~V} / \mu \mathrm{m}$, where $m$ is a material parameter ranging from less than 1.0 to greater than 3.0.

25. Both approximations were justified physically ${ }^{6,19}$ in terms of the inequality $E_{d} \ll E \ll E_{q}$, where $E_{q}$ and $E_{d}$ are the limiting space-charge field and the diffusion field, respectively, evaluated at the soliton width of no less than $5 \mu \mathrm{m}$.

26. $\left\langle\cos ^{m} \theta\right\rangle=\int_{0}^{\pi} \cos ^{m} \theta \exp \left(-U / k_{B} T_{a}\right) \sin \theta \mathrm{d} \theta / \int_{0}^{\pi} \exp \left(-U / k_{B} T_{a}\right)$ $\times \sin \theta \mathrm{d} \theta$, where $U=-\mu_{\mathrm{D}} \cdot \mathbf{E}^{0}-\mathbf{p} \cdot \mathbf{E}^{0} / 2$ is the dipole interaction energy, $k_{B}$ is the Boltzmann constant, and $T_{a}$ is the ambient temperature. We can safely neglect the induced dipole energy, $\mathbf{p} \cdot \mathbf{E}^{0} / 2$, since for typical photorefractive polymers ${ }^{22}$ with the orientational enhancement photorefractive effect, $\mu_{D} \cong 10 \mathrm{D}$ and $\Delta \alpha^{0}=5 \times 10^{-23} \mathrm{~cm}^{3}$, under the dc field $E<100 \mathrm{~V} / \mu \mathrm{m}$, the induced dipole energy is less than $1 / 60$ of the total energy $U$. Thus $U \cong-\mu_{D} \cdot \mathbf{E}^{0}=-\mu_{D} E \cos \theta$.
27. A. Galvan-Gonzalez, M. Canva, G. Stegeman, R. Twieg, K -P. Chan, T. Kowalczyk, X. Zhang, and H. Lackritz, "Systematic behavior of electro-optic chromophore photostability," Opt. Lett. 25, 332-334 (2000).

28. C. Moylan, R. Wortmann, R. Twieg, and I. McComb, "Improved characterization of chromophores for photorefractive applications," J. Opt. Soc. Am. B 15, 929-932 (1998).

29. C. Chen and S. Chi, "Subwavelength spatial solitons of TE mode," Opt. Commun. 157, 170-172 (1998).

30. J. A. Herlocker, K. B. Ferrio, E. Hendrickx, B. D. Guenther, S. Mery, B. Kippelen, and N. Peyghambarian, "Direct observation of orientation limit in a fast photorefractive polymer composite," Appl. Phys. Lett. 74, 2253-2255 (1999).

31. D. Wright, M. A. Diaz-Garcia, J. D. Casperson, M. DeClue, W. E. Moerner, and R. J. Twieg, "High-speed photorefractive polymer composites," Appl. Phys. Lett. 73, 1490-1492 (1998).

32. K. S. West, D. P. West, M. D. Rahn, J. D. Shakos, F. A Wade, K. Khand, and T. A. King, "Photorefractive polymer composite trapping properties and a link with chromophore structure," J. Appl. Phys. 84, 5893-5899 (1998).

33. K. D. Singer, J. E. Sohn, and S. J. Lalama, "Second harmonic generation in poled polymer films," Appl. Phys. Lett. 49, 248-250 (1986). 